\title{
Transient Probabilities for Queues with Applications to Hospital Waiting List Management
}

\author{
MARK JOY* \\ School of Mathematics, Kingston University \\ E-mail: m.joy@kingston.ac.uk \\ SIMON JONES \\ Bromley NHS
}

\begin{abstract}
In this paper we study queuing systems within the NHS ${ }^{1}$. Recently imposed government performance targets lead NHS executives to investigate and instigate alternative management strategies, thereby imposing structural changes on the queues. Under such circumstances, it is most unlikely that such systems are in equilibrium. It is crucial, in our opinion, to recognise this state of affairs in order to make a balanced assessment of the role of queue management in the modern NHS. From a mathematical perspective it should be emphasised that measures of the state of a queue based upon the assumption of statistical equilibrium (a pervasive methodology in the study of queues) are simply wrong in the above scenario. To base strategic decisions around such ideas is therefore highly questionable and it is one of the purposes of this paper to offer alternatives: we present some (recent) research whose results generate performance measures and measures of risk, for example, of waiting-times growing unacceptably large; we emphasise that these results concern the transient behaviour of the queueing model-there is no asssumption of statistical equilibrium. We also demonstrate that our results are computationally tractable.
\end{abstract}

Keywords:

\section{Introduction}

This paper presents theory whose results we apply to the flow of trauma and orthopaedic elective patients at a Bromley ${ }^{2}$ hospital over the period 1/10/2001-5/3/2004. We model this activity in a fairly crude way as a pure immigration-emigration system, experiencing Poisson arrivals and departures. To be precise, we abstract the waiting list for these patients as a queue, we form frequency histograms of arrivals and departures from the list and fit Poisson models. We make no attempt to model the patients' length of stay in hospital in any sophisticated way.

Clearly there are gross estimates implicit in such a modelling approach and we will discuss these in more detail in Section 7, but our purpose in such a methodology is to make an attempt at answering specific strategic questions such as: is it possible for such an acute hospital to service up to the 80-th percentile of its offered load? This is an oft-quoted maxim of good practice (particularly by the NHS Modernisation Agency) and has the status of folklore: the authors are unable to find references in the literature of queueing theory affirming such a strategy. In this paper we hope to show that achieving such a performance target is more or less impossible given the current capacity of this hospital (see Section 6).

\footnotetext{
${ }^{*}$ Corresponding author.

${ }^{1}$ The reader should note that NHS stands for National Health Service, the state provider of health care in the UK.

${ }^{2}$ A SE suburb of London, UK.
}

Further interesting questions arise in an obvious way: what is the probability (given the current state of a queue) that, by time $t, n$ customers are serviced? Moreover, we may wish to study what happens to such probabilities if there is a change in the system parameters. Suppose, for example, that we introduce intermediate treatment centres-directly affecting the arrival rate-what effect will this have on such chances?

Of central importance to NHS managers is the question: with what chance can the new Government targets be satisfied? Or to put this is a more realistic way, we may ask what magnitude changes need to be imposed in order to satisfy the new Government targets to within a high probability? We may also frame the previous questions with this proviso, that is we can answer such questions with a risk $r$ attached and insist that $r$ is suitably small.

Throughout this paper we employ the theory of $M^{b} / M / 1$ queues, that is, we utilise results concerning single-server queues with Poisson arrivals (possibly in batches of size $b>1$ ) with parameter $\lambda$ and independent, exponentially distributed service times (with mean $\frac{1}{\mu}$ ). However, we emphasise again that we make no assumption on the traffic intensity to ensure steady state equilibrium, thus we are interesting in the transient behaviour of an $M^{b} / M / 1$ queue and we draw upon the theory presented for example in [1-3].

In the next section we briefly outline this theory and present an important result from this theory in Section 3. In Section 4 we propose three performance measures, tools which capture the risks associated with certain of the above questions. In Section 5 we use the data set of arrivals and departures to estimate parameters, then in Section 6 we apply our theory to 


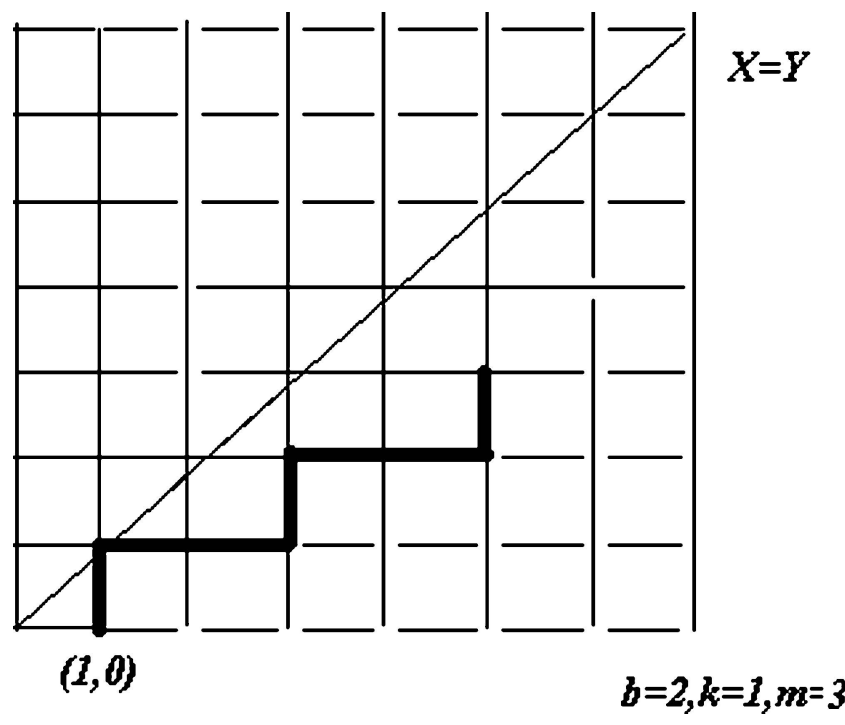

Figure 1. A lattice path.

investigate the above questions. Finally, in Section 7 we discuss the future direction this research may take.

\section{2. $M^{b} / M / 1$ Queues: State space, markov assumptions \& path counting}

In this section we will briefly outline the relevant theory from [1], assuming batch arrivals of size $b$ and that $k$ customers are present in the queue initially. The reader should be aware that $b=1$ is admissible, thus we deal with the classical case of single arrivals at the same time. Now consider the time interval $[0, t]$ and divide into a number of slots of duration $h>0$, such that $t / h \in \mathbb{N}$, where $\mathbb{N}$ is the set of natural numbers. Assume $h$ is so small that

- No more than one batch may arrive in a time slot

- No more than one customer may depart in one time slot

- These are mutually exclusive events

- Events in different time slots are independent.

We represent an $M^{b} / M / 1$ queue on a lattice, $L,(L=$ $\{(x, y) \mid x, y \in \mathbb{N}\})$ in the plane: at any moment in time we map the state onto the point $(x, y) \in L$, where $(x-k) / b$ batches have arrived and $y$ departures have taken place.

It is here that the utility of such a representation of a queue becomes immediately apparent for we are able to track the evolution of the queue on $L$. We start at $(k, 0)$, which clearly represents the initial state of the system with $k$ customers waiting to be serviced, and walk to $(m, n)$, taking horizontal steps of size $b$ and vertical steps of size 1 . Thus we impose the following representation: vertical segments of $L$ represent departures from the queue whilst horizontal segments represent arrivals into the system. Figure 1 represents such an evolution, consisting of 3 departures, 2 arrivals with $b=2, k=1$.
The following table attaches probabilities to transitions along $L$.

\begin{tabular}{llll}
\hline From $(x, y)$ to: & $(x+b, y)$ & $(x, y+1)$ & $(x, y)$ \\
\hline Busy & $\alpha$ & $\beta$ & $\gamma$ \\
Idle & $\alpha$ & $o(h)$ & $\delta$ \\
\hline
\end{tabular}

where,

$$
\begin{aligned}
& \alpha=\lambda h+o(h), \\
& \beta=\mu h+o(h), \\
& \gamma=1-\lambda h-\mu h+o(h), \\
& \delta=1-\lambda h+o(h) .
\end{aligned}
$$

Here, $o(h)$ stands for any function of $h$ satisfying $\lim o(h) / h=0, h \rightarrow 0$.

Later in this paper we will have occasion to refer to the busy period of a queue. Quite simply a busy period is a time during which the server is never idle, during this period when a customer finishes service there will be a new one waiting for attention. Conversely an idle period commences when no customers are left in the system.

Now in order to track the evolution we will need to be able to count lattice paths, that is paths in the plane consisting of vertical and horizontal segments of $L$. There are many classical results concerning the enumeration of such paths, see for example [4-6]. (The reader should note that these references provide counting methods for polygonal paths: these are lattice paths that have been rotated by $-\pi / 4$ ).

As a flavour of the type of results on lattice path counting we offer the following simple observations. The total number of paths from $(k, 0)$ to $(b m+k, n)$ is given by

$$
\left(\begin{array}{c}
m+n \\
n
\end{array}\right)
$$

(see [1], Result 1). This is a simple application of Pascal's Triangle, it tells you how many routes there are (in a city whose streets are a rectangular grid) from one intersection of streets to another such intersection. Of vital importance is the following result: the number of paths not touching $X=Y$ is

$$
\frac{k}{(b+1) m+k}\left(\begin{array}{c}
(b+1) m+k \\
m
\end{array}\right) \text {. }
$$

(see [1], Result 2).

The reason for the importance of this result is the physical significance it carries, once a path has reached the line $X=Y$ in the plane an idle period begins since there are no customers left at this point, thus $X=Y$ represents a barrier which evolutionary paths cannot cross. This is the reason for the entries, $o(h)$, in table 1 , since meeting the barrier $X=Y$ corresponds to entering an idle period.

In what follows we use the following notation: $T_{c}(k ;, m, n)$ is the number of paths from $(k, 0)$ to $(b m+k, n)$, which touch or cross the barrier $X=Y ;[x]$ represents the integer part of $x$. 
We have the following formula for $T_{c}$.

$$
\begin{aligned}
T_{c}= & \sum_{r=0}^{\left[\frac{(n-k)}{b}\right]} \frac{k}{(b+1) r+k}\left(\begin{array}{c}
(b+1) r+k \\
r
\end{array}\right) \\
& \times\left(\begin{array}{c}
m+n-(b+1) r-k \\
n-b r-k
\end{array}\right) .
\end{aligned}
$$

(see, [1], Eq. (4), p. 335).

\section{Transient probabilities}

In this section we present three results (without proof) from the theory presented in $[1,2]$. These results have direct relevance to the type of questions we posed in the introduction but are a small subset of the impressive array of computational results available from the lattice path counting approach.

Firstly, we present a formula for the probability that the queue starting initially with $k>0$ units attains queue length $b m+k-n$ at time $t$, encountering arrivals of $m$ batches and providing $n$ services. Such a formula settles many questions concerning the evolution of the queue over the time interval $[0, t]$.

If $P_{m, n ; k}^{b}(t)$ denotes the above probability we have the important result.

Theorem 3.1. If $b m+k-n \geq b$ then

$$
\begin{aligned}
P_{m, n ; k}^{b}(t)= & e^{-(\lambda+\mu) t}(\lambda t)^{m}(\mu t)^{n} \cdot\left(\left(\begin{array}{c}
m+n \\
n
\end{array}\right)-T_{c}(k ; m, n)\right) \\
& -e^{-\lambda t}\left(\frac{\lambda}{\mu}\right)^{m} \sum_{j=1}^{\left[\frac{(n-k)}{b}\right]+1}\left(T_{c}(k+(j-1) b ; m, n)\right. \\
& \left.-T_{c}(k+j b ; m, n)\right) E_{j-1, m+n}(\mu t),
\end{aligned}
$$

where,

$$
\begin{aligned}
E_{i, j}(\theta)= & \sum_{k=0}^{i} \frac{(-1)^{k}}{(i-k) !}\left(\begin{array}{c}
k-1+j-i \\
k
\end{array}\right) \\
& \times \frac{\left(1-\sum_{l=1}^{j-i+k-1} e^{-\theta} \frac{\theta^{l}}{l !}\right)}{\theta^{k-1}} .
\end{aligned}
$$

There is a similar formula for the case given by $0<b m+k-$ $n<b$. For a proof of this result see [2], Theorem 4 .

Whilst algebraically the above result is complex it does provide a computationally tractable performance measure. Specifically, it provides us with an answer to a strategic question such as: given the current state of the queuing system (or even given an hypothesised current state) what is the probability of servicing a given number of customers in a given time period? This has usefulness both in studying the current system and also in answering what-if type of questions. For example, suppose we tune the system by providing more beds (perhaps another ward is brought onto line), perhaps we employ more consultants, or at any rate increase the 'capacity' of the system, then provided we can measure the effect that this increased capacity has on the parameters $\lambda$ and $\mu$, we will be able to understand the global effect on the waiting list.

Let $P_{n ; k}(t)$ denote the probability that the first busy period still persists by time $t>0$, with $n$ customers in the queue (when initially there were $k$ ).

Theorem 3.2. For $k>0$ we have

$P_{n ; k}(t)=e^{-(\lambda+\mu) t}\left(\frac{\lambda}{\mu}\right)^{\frac{(n-k)}{2}}\left(I_{n-k}(2 \sqrt{\lambda \mu} t)-I_{n+k}(2 \sqrt{\lambda \mu} t)\right)$,

where $n \geq 1$ and $I_{k}(x)$ is the modified Bessel function of the first kind (for a discussion of Bessel functions, see [7]).

The last result that we present here concerns the number of customers served in the busy period. We have

Theorem 3.3. The probability that $m$ batches complete their service during a busy period of arbitrary duration is given by

$$
\frac{1}{m}\left(\begin{array}{c}
(b+1) m-2 \\
m-1
\end{array}\right)\left(\frac{\lambda}{\lambda+\mu}\right)^{m-1}\left(\frac{\mu}{\lambda+\mu}\right)^{b m}
$$

It is worth mentioning that even if the queue possesses a steady state $(\lambda<\mu)$ and stability is the order of the day we will still be interested in the results presented in this section for they answer fundamental questions concerning the shortto medium-term behaviour of the waiting list. If $\lambda \geq \mu$ and no such equilibrium exists, arguably we are even more interested in such formulae.

\section{Performance measures}

In this section we propose to introduce three performance measures directly linked to the results of the previous section. Perhaps the simplest measure is the number of customers that we are able to service. To this end let $P_{t}^{b}(n)$ be the probability that $n$ customers are served by time $t$ in the face of batch arrivals of size $b$.

Using the results from Section 3 it is clear that

$$
P_{t}^{b}(n)=\sum_{m=\left[\frac{(n-k)}{b}\right]}^{\infty} P_{m, n ; k}^{b}(t)
$$

If we were immune to time constraints we may wish to introduce the measure, $Q_{j}^{b}$, the probability given by Theorem 3.3. Essentially we are then interested in measuring the likelihood of servicing $m$ batches during a busy period.

$$
Q_{j}^{b}(t)=\frac{1}{j}\left(\begin{array}{c}
(b+1) j-2 \\
j-1
\end{array}\right)\left(\frac{\lambda}{(\lambda+\mu)}\right)^{j-1}\left(\frac{\mu}{(\lambda+\mu)}\right)^{b j}
$$

This measure is likely to be more useful in specialising our reseach to study admissions to one clinical stream or to outpatients' clinics, rather than the global modelling attmept presented in this paper. In such cases busy periods have a direct relevance to system performance. When studying the aggregated waiting list for all disciplines, it is possible that there are 


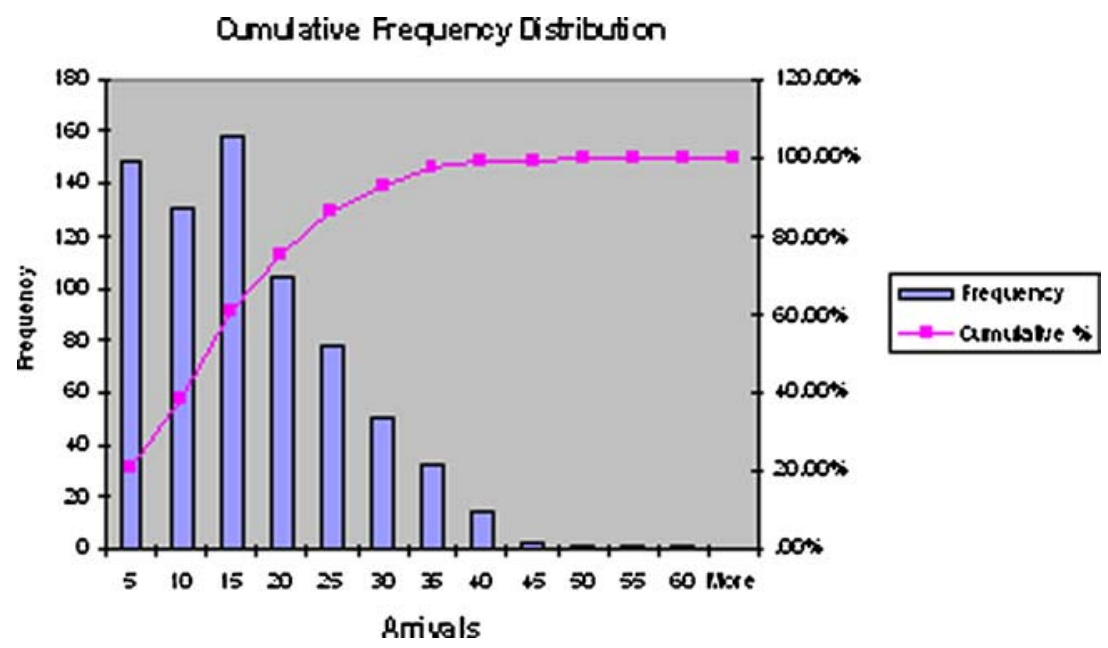

Figure 2. Arrivals at Bromley hospital 1/10/2003-5/3/2004.

times when $\lambda>\mu$ and busy periods have positive probabilities of being permanent! How many customers are served in the entire history of the hospital is of course not relevant to the questions we have posed in this paper.

Finally we propose the following as a performance measure

$$
R_{n}(t)=e^{-(\lambda+\mu) t}\left(\frac{\lambda}{\mu}\right)^{\frac{(n-k)}{2}}\left(I_{n-k}(2 \sqrt{\lambda \mu} t)-I_{n+k}(2 \sqrt{\lambda \mu} t)\right)
$$

After Theorem 3.2 we see that Eq. (3) gives probabilities for various queue sizes. In order to compute $R_{n}(t)$ it is important to note that modified Bessel functions are provided as native functions in symbolic computational packages such as MAPLE $^{\mathrm{TM}}$.

\section{Application to Bromley hospital}

Frequency histograms for the arrivals at Bromley hospital between the period 1/10/2001-5/3/2004 were compiled (see figure 2) and a Poisson distribution with mean 2.289 was fitted. A $\chi^{2}$ test for goodness-of-fit at the 5\% level of significance provided no evidence to reject this model. Here of course we are replacing the regular day-to-day arrival mechanism of such patients by an assumed random arrival pattern. This is, of course, a weakness in the modelling. In our discussion of future directions for this research (see Section 7) we indicate that perhaps a more precise application of the ideas of this paper would occur if we attempted to model arrivals into one clinical stream, rather than adopting the global approach taken here.

As we have previously stated we use departures from the waiting list for trauma and orthopaedic patients as a proxy for the service completions of these patients. Again this is a potential weakness of this the globally constructed model. Thus we are embedding a Markov model at the time instants of service-completion, measured by departure from the list; this is a common device in queuing theory. Implicit of course is the assumption that departure from the list is equivalent to admittance to the hospital.

For the departures (see figure 3) we fitted a Poisson with a mean of 1.639 . The $\chi^{2}$-statistic for the goodness-of-fit was well within the non-critical region at the $5 \%$ level for 8 degrees of freedom.

We discuss in more detail the shortcomings and possible improvements in our methodology in Section 7.

\section{Performance curves at Bromley hospital}

In this section we study the performance measure, $R_{n}(t)$, see Section 4, Eq. (3), that directly addresses one of the questions raised in the introduction, namely managing sufficient capacity to discharge up to the 80th percentile of offered load. One of the authors attended an NHS Modernisation Agency workshop where such a recommendation was presented as good practice. For Bromley Hospital this would imply that 22 people/day are serviced, i.e. leave the queue.

In the dimensionless units that we have chosen to fit our models we can plot the following performance curves for $R_{n}(t)$, indicating the effect of adding extra capacity into the system as measured by an increasing $\mu$. Figure 4 was constructed in the following way. At the last date in our data set we measured the size of the waiting list. With the estimated values of $\lambda$ and $\mu$ we ran the queue foward in time assuming that we were servicing the 80th percentile and experiencing arrivals according to our Poisson model, the queue size is then iterated into the future assuming this behaviour.

The inner curve in figure 4 shows probabilities given current (estimated) values of $\lambda$ and $\mu$, the outermost curve shows the effect on these probabilities if $\mu$ is increased to one and a half times the current estimated value of $\lambda$; the middle curve indicates the case $\lambda=\mu$. The reader can see (albeit rather crudely) the important efect that increasing $\mu$ has; also one can see how unlikely, given current capacity (in terms of $\mu$ ) one is able to service such an onerous target. 


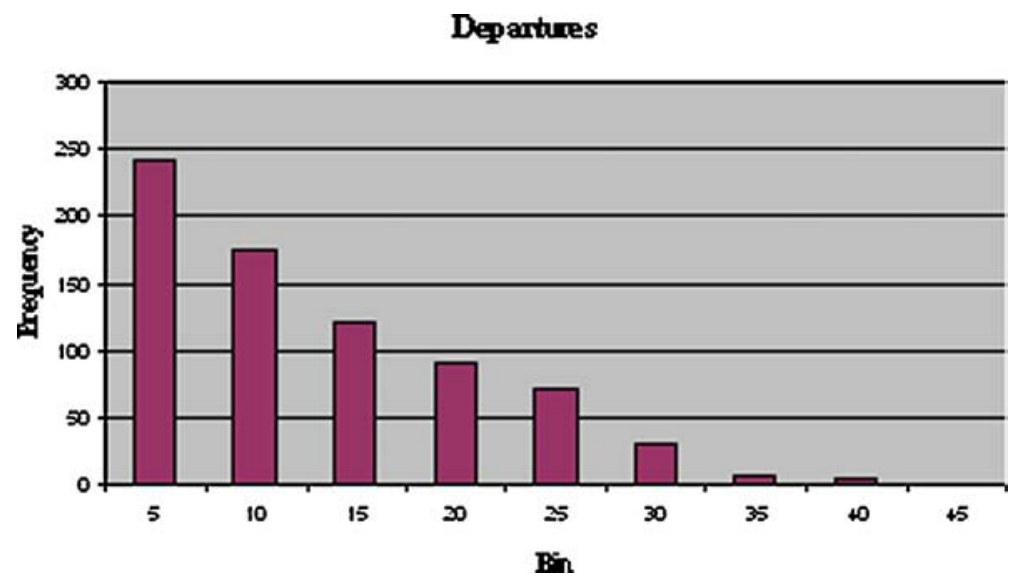

Figure 3. Departures from the waiting list at Bromley hospital 1/10/2001-5/3/2004.

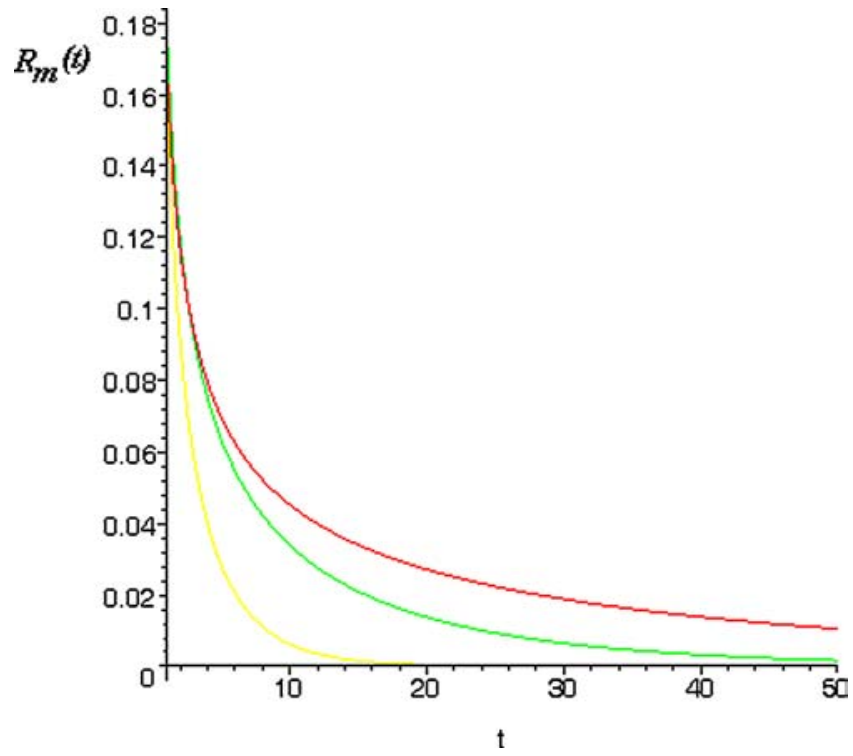

Figure 4. Throughput at Bromley hospital.

These computations were performed by MAPLE $8^{\mathrm{TM}}$ running on a PENTIUM 4 platform in approximately 3.9 seconds.

Clearly, by introducing extra capacity into the system resulting in either a reduced $\lambda$ or an increased $\mu$ we increase our chances of being meeting such a target. However, even in a highly efficient system where $\mu>\frac{3}{2} \lambda$, the probability of servicing and thereby discharging this number of people every day quickly shrinks to less than a $5 \%$ chance over a time horizon of approximately 10 days!

\section{Discussion}

Across the NHS, trauma and orthopaedic arrival and departure patterns are notoriously difficult to model and we have modelled in a crude way such activity. There are shortcomings in the methodology that cast some doubt on the current findings and applicability of this work. In particular we intend to model more closely the service mechanism for this stream of patients in an effort to answer the question of how closely our model follows the real world system it purports to study. Our current method of indirectly measuring this distribution by observing hospital admittance and thus departures from the waiting list needs replacing with a finer analysis of the service-time distribution, possibly for a given stream of patients. We also intend to model out-patient clinics in this way, here service mechanisms are easier to model. In spite of these difficulties though, we contend that our approach provides a useful tool in evaluating the order of difficulty in achieving certain targets.

We also need to find ways of measuring the effect of capacity changes on the parameters $\lambda$ and $\mu$. In an effort to increase the capacity of their system Bromley Hospital has recently (as of December 2003) introduced a treatment centre which captures and treats a fast-track of patients that otherwise would have contributed to elective admissions. Preliminary results indicate the effectiveness in reducing the waiting list for this hospital. Clearly the data set on which we have estimated our parameters does not capture this activity and so it would be unrealistic to expect a tight correspondence between the list management in its current state and our model. From our point of view it is important to measure the change in $\lambda$ that such innovations effect and indeed this is a way forward for this research. If one can reliably measure the effect of such capacity changes (globally or on a given stream) we can make robust predictions concerning the future state of the waiting list. Incidentally, such structural changes mean that the queuing mechanism will certainly not be in equilibrium at Bromley.

One further strand of this research is provided by the following result concerning the distribution of maximum waiting time $w^{*}$ at time $t$, given $k>0$ initial customers,

$$
P_{t}\left(w^{*} \leq W \mid k\right)=\sum_{\substack{n, s \\ b \mid n+s-k}}^{\infty} P_{\left(\frac{n+s-k}{b}\right), s ; k}(t) \cdot P\left(t-\xi_{\left[\frac{n}{b}\right]+1} \leq W\right)
$$

$\xi_{k}$ is the arrival time of the $k$ th batch (for a proof of this result see [3]). Poisson arrivals imply that the second term on the right hand side of this equation is a Gamma distribution, so we can compute such a probability. This result provides us with a way of tracking the maximum waiting time (assuming a first-come-first-serve discipline) in NHS queues. 
Targets are here to stay. Indeed the current government made election pledges to reduce number of people waiting for NHS service across the UK: for example, from 31/3/2004 the Government require hospitals to reduce maximum waiting time (for in-patients) from 12 months to 9 months. It is our contention that the methods of this paper provide useful performance measures of the effectiveness of queue management within the NHS and are useful as a means of assessing the likelihood of meeting such targets.

\section{References}

[1] K. Sen and J.L. Jain, Combinatorial approach to Markovian queueing models, J. Statistical Plann. and Inference 34 (1993) 269-279.
[2] K. Sen and R. Gupta, Transient solution of $M / M / 1$ queues with batch arrival: A new approach, Statistica 3 (1996) 333343.

[3] Mark Joy, Transient analysis of the waiting times in $M / M / 1$ queues (Submitted for publication, 2004).

[4] W. Feller, An Introduction to Probability Theory and Its Applications, Vol 2 (Wiley \& Sons, New York, 1971).

[5] S.G. Mohanty, The distribution of the maximum queue length, the number of customers served and the duration of the busy period for the queueing system $M / M / 1$ involving batches, INFOR, 9, 1971.

[6] L. Takács, Combinatorial Methods in the Theory of Stochastic Processes (John Wiley \& Sons, New York, 1967).

[7] Milton Abramowitz and Irene A. Stegun, Handbook of Mathematical Functions with Formulas, Graphs, and Mathematical Tables (Dover Publications, Inc., New York, 1970). 